\title{
White matter hyperintensities and self-reported depression in a sample of patients with chronic headache
}

\author{
Gianluca Serafini • Maurizio Pompili • Marco Innamorati • Andrea Negro • \\ Martina Fiorillo - Dorian A. Lamis - Denise Erbuto - Francesco Marsibilio • \\ Andrea Romano • Mario Amore · Lidia D'Alonzo • Alessandro Bozzao • \\ Paolo Girardi · Paolo Martelletti
}

Received: 30 August 2012/ Accepted: 10 October 2012/Published online: 19 October 2012

(C) The Author(s) 2012. This article is published with open access at Springerlink.com

\begin{abstract}
White matter hyperintensities (WMH) have been associated with mood disorders in psychiatric patients. In the present study, we aimed to assess whether WMHs are associated with depressive symptoms and different sensitivity of the behavioral inhibition (BIS), and activation (BAS) systems in patients with chronic headache. Participants were 85 adult outpatients (16 men and 69 women) with a diagnosis of chronic headache. All of the
\end{abstract}

G. Serafini · M. Pompili ( $₫) \cdot$ M. Innamorati · D. Erbuto · P. Girardi

Department of Neurosciences, Mental Health and Sensory Organs, Suicide Prevention Center, Sant'Andrea Hospital, Sapienza University of Rome, 1035-1039,

Via di Grottarossa, 00189 Rome, Italy

e-mail: maurizio.pompili@uniroma1.it

A. Negro · M. Fiorillo · F. Marsibilio · L. D’Alonzo ·

P. Martelletti

Department of Clinical and Molecular Medicine,

Sapienza University of Rome and Regional Referral

Headache Centre, Sant'Andrea Hospital, Rome, Italy

A. Negro

Department of Radiology, Stroke and Neurovascular Regulation Lab, Harvard Medical School, Massachusetts General Hospital, Boston, MA, USA

D. A. Lamis

Department of Psychiatry and Behavioral Sciences, Emory University School of Medicine, Atlanta, GA, USA

A. Romano $\cdot$ A. Bozzao

Division of Neuroradiology, Department of Neuroscience, Sant'Andrea Hospital, Sapienza University of Rome,

Rome, Italy

M. Amore

Department of Neuroscience, Rehabilitation, Ophthalmology, Genetics, Maternal and Child Health, Section of Psychiatry, University of Genova, Genova, Italy patients underwent brain magnetic resonance imaging (MRI) and were administered the BIS/BAS scales and the Center for Epidemiologic Studies Depression Scale. Above $40 \%$ of patients had periventricular WMHs (PWMHs) and almost $98 \%$ had deep WMHs (DWMHs). Patients with PWMHs reported fewer depressive symptoms than patients without PWMHs. Patients with more severe DWMHs (compared with patients with mild or without DWMH lesions) were older and reported lower scores on the drive dimension of the BIS/BAS scales. In multivariate analyses, patients with PWMHs were 1.06 times more likely to report fewer depressive symptoms than patients without PWMHs. WMH lesions in patients with chronic headache were associated with less depression severity.

Keywords PWMHs - DWMHs - Chronic headache . Self-reported depression $\cdot$ Age

\section{Introduction}

White matter hyperintensities (WMHs) appear as hyperintense signals on T2-weighted magnetic resonance images (MRI) and represent ependymal loss and differing degrees of myelination in the brain [1,2]. WMHs, depending on the localization, are commonly classified as periventricular white matter hyperintensities (PWMHs) and deep white matter hyperintensities (DWMHs). WMHs have been reported to be commonly associated with older age and cardiovascular risk factors such as hypertension and diabetes [3-5]. Degenerative changes in brain white matter have been reported to be associated with mood disorders and suicidal behavior both in children and young adults [6-13]; however, they seem not to be specific to first episode psychotic disorders [14]. Taylor and colleagues 
hypothesized that patients with WMHs may be at a higher risk for suicide and developing mood disorders due to a possible disruption of neuroanatomic pathways [15]. Mood regulation depends on the complex extensive connections between the prefrontal cortex, amygdala-hippocampus complex, thalamus and basal ganglia [16].

Studies in both clinical and community-based settings have demonstrated the association between migraine and a number of specific psychiatric disorders, particularly major affective disorders [17-23]. Previous researchers have hypothesized that the relation between migraine and psychiatric disorder may be bi-directional given that migraine patients have a more than threefold risk of developing depression and the presence of depression is also associated with a greater risk of developing a migraine [21, 24].

Since the 1990s, brain MRI studies have investigated the presence of focal hyperintense abnormalities in patients with migraine [25]. Chronic migraine has been associated with the presence of white matter hyperintensities and migraine attack frequency, and attack history may be considered as a contributor of brain abnormalities in migraineurs [25]. In a sample of 28 patients with migraine, using high-resolution T1- and diffusionweighted MRI and optimized voxel-based morphometry to localize gray and white matter density, Schmitz et al. [26] found that regions of brain abnormalities in migraineurs were mainly located in the frontal lobes, brainstem, and the cerebellum, and both attack frequency and disease duration predicted brain damage in migraine patients. To date, although the cause of migraine, particularly with aura, is commonly recognized as ischemic, which is consistent with its association with vascular risk factors [25], the natural history of white matter abnormalities in migraine patients is not completely understood. The relation between increased volume of white matter hyperintensities and a history of severe headache is also a matter of debate.

The structures and functions of a major motivational system, the Behavioral Inhibition System (BIS), have been described by Gray [27] in 1994. It has been suggested that the BIS modulates behavioral inhibition, anxiety, attention, and arousal towards signals of fear stimuli and punishment $[28,29]$. A strong BIS is indicative of anxiety and inhibition even if few fear stimuli are present, whereas a weak BIS may not contribute to anxiety and inhibition even when severe stimuli occur. Gray [27, 28] also described the behavioral activation system (BAS) as a regulatory system measuring positive affect and approach behavior towards signals of reward. BAS activity may trigger positive affect and increase the likelihood of incentive acquisition. Refractoriness to incentives, low positive affect, and an absence of environmental engagement may be associated with a weak BAS [30].

As suggested by Gray [27, 28], the BIS may be considered an anxiety system and its activity may be investigated in several psychiatric conditions [31]. The BIS scale [32] includes seven items and assesses the tendency to respond with negative affect, anxiety, or fear in response to threatening events. The BAS scales [32] describe the tendency to respond with positive affect and motivation when faced with incentives or rewards. To our knowledge, no studies have investigated the sensitivity of the BIS and activation of the BAS concurrently with self-reported depression among patients with WMHs and chronic migraine.

In the present study, we aimed to assess whether WMHs are associated with self-reported depressive symptoms and different sensitivity of the BIS and BAS systems in patients with chronic headache.

\section{Methods}

\section{Participants}

Participants were 85 adult outpatients (16 men and 69 women) admitted at the Regional Referral Headache Centre, Department of Medical and Molecular Sciences, Sapienza University of Rome, Italy, between June 2010 and June 2011. Inclusion criteria were 18 years of age or older and a diagnosis of primary headache. Exclusion criteria included the presence of any psychiatric disorders or any condition affecting the ability of the patient to complete the assessment, including the denial of informed consent. The mean age of the sample was $50.13 \pm 12.83$ years (min/max 20/88 years). Table 1 summarizes the headache diagnoses as per the international classification of headache disorder (ICHD-II, ICHD-IIR).

All the patients voluntarily participated in the study and provided written informed consent. The design of the study was approved by the Hospital's Ethics Committee.

Measures: clinical assessment

All the patients were administered the BIS/BAS scales and the Center for Epidemiologic Studies Depression Scale (CES-D).

\section{BIS/BAS scales}

The BIS scale [32] includes seven items and measures the tendency to respond with negative affect, anxiety, or fear in response to threatening events. An example of an item on 
Table 1 Diagnoses in the 85 study subjects (ICHD-II, ICHD-IIR)

\begin{tabular}{lcc}
\hline & Frequency $^{\mathrm{a}}$ & $\%$ \\
\hline Chronic migraine & 69 & 81.1 \\
Migraine without aura & 12 & 14 \\
Migraine with aura & 7 & 8.2 \\
Episodic tension-type headache & 4 & 4.7 \\
Chronic tension-type headache & 3 & 3.5 \\
\hline
\end{tabular}

${ }^{a}$ Some patients presented multiple diagnoses

the BIS is "If I think something unpleasant is going to happen I usually get pretty 'worked up'." The BAS scales [32] describe the tendencies to respond with positive affect and motivation when faced with incentives or rewards. The BAS includes three distinct subscales: reward responsiveness, drive, and fun seeking. Individuals scoring high on the drive subscale are strongly motivated towards specific goals. The tendency to respond with elevated energy and positive affect when desired events are experienced was measured by the reward responsiveness scale. The fun-seeking subscale assessed the impulsive behavioral desire of fun conditions. Adequate psychometric properties (internal consistency, factor structure, test-retest reliability) of the BIS/BAS scales have been demonstrated [32-34]. Normative data were derived by a major community sample [34].

\section{CES-D scale}

The CES-D scale is a short self-report scale designed to measure current level of depressive symptomatology in the general population, with an emphasis on the affective component. The 20 items were selected from previously validated depression scales. Participants were asked to rate each item on a scale from 0 (rarely or none of the time: less than 1 day) to 3 (most or all of the time 5-7 days) on the basis of how often they have felt that way during the previous week. The validity of the scale as a screening tool for depression has been documented in several populations [35-42]. In our sample, the Cronbach alpha for the CES-D was 0.86 .

Magnetic resonance image acquisition and rating of white matter hyperintensities

Brain MRIs were performed using a Siemens Sonata equipment, Erlangen, Germany (1.5 T). FLAIR scan sequence was used for WMH measurement (ax: TR 10000; TE 125; thickness $5 \mathrm{~mm}$; matrix $144 \times 256$ ). Proton density and $\mathrm{T} 2$-weighted images were obtained (PD and $\mathrm{T} 2$ ax: TR 2870; TE 13/107; thickness $5 \mathrm{~mm}$; matrix $147 \times 256$ ) in the axial and the coronal planes. Axial and sagittal
Table 2 DWMHs and PWMHs as assessed by the Fazekas modified scale

\begin{tabular}{lcc}
\hline & PWMHs & DWMHs \\
\hline 0 & 57.6 & 2.4 \\
1 & 30.6 & 56.5 \\
2 & 8.2 & 34.1 \\
3 & 3.5 & 7.1 \\
\hline
\end{tabular}

T1-weighted images were also obtained (T1 ax: TR 647; TE 17; thickness $5 \mathrm{~mm}$; matrix $128 \times 192 \mathrm{~T} 1$ sag: TR 552; TE 17; thickness $5 \mathrm{~mm}$; matrix $231 \times 192$ ). The presence of WMH was assessed by a neuroradiologist blind to all clinical information, using the modified Fazekas fourpoint rating scale which describes MRI hyperintensities on an ascending scale of intensity and frequency [43]. A second neuroradiologist, blind to all clinical information and previous ratings, reviewed all MRI films. The mean $k$ value for interrater reliability for both PWMHs and DWMHs was 0.90. DWMHs and PWMHs as assessed by the Fazekas modified scale are summarized in Table 2.

Statistical analysis

A series of $t$ tests and one-way Fisher exact tests were used to assess differences between groups. All the variables significant at the bivariate analyses were inserted as independent variables in a generalized linear model with a robust estimator. Groups were inserted as criterion. Associations between variables are reported as odds ratios (OR). All of the analyses were performed with the statistical package for social sciences SPSS 19.0 for Windows.

\section{Results}

Above $40 \%$ of patients had PWMHs and almost $98 \%$ had DWMHs (see Table 2). Due to the low number of participants included in some categories, we collapsed levels of severity of white matter hyperintensity into two classes: (1) periventricular lesions were categorized in absent/ present; and (2) deep lesions assessed as 0 and 1 at the Fazekas were collapsed together while levels 2 and 3 were combined.

Patients with PWMHs differed from those without periventricular lesions on depression severity $\left(t_{77.76}=\right.$ 2.30; $P<0.05$ ) (Table 3). Patients with PWMHs had lower CES-D scores $(13.79 \pm 7.51$ vs. $18.19 \pm 9.68)$ than patients without PWMHs. Groups did not differ in age $(P=0.37)$, sex $(P=0.17)$, or for scores on the BIS/BAS scales. 
Table 3 Differences between groups

\begin{tabular}{|c|c|c|c|c|c|c|c|c|}
\hline & \multicolumn{4}{|l|}{ PWMHs } & \multicolumn{4}{|l|}{ DWMHs } \\
\hline & $\begin{array}{l}\text { Absence of } \\
\text { PWMHs }\end{array}$ & $\begin{array}{l}\text { Presence of } \\
\text { PWMHs }\end{array}$ & Test & $P$ & $\begin{array}{l}\text { No DWMHs or } \\
\text { mild lesions }\end{array}$ & $\begin{array}{l}\text { Moderate to severe } \\
\text { DWMHs lesions }\end{array}$ & Test & $P$ \\
\hline Men & $14.3 \%$ & $25.0 \%$ & & $<0.17$ & $20.0 \%$ & $17.1 \%$ & & $<0.48$ \\
\hline Age & $49.02 \pm 12.76$ & $51.58 \pm 12.96$ & $t_{83}=-0.90$ & $<0.37$ & $47.40 \pm 11.91$ & $53.89 \pm 13.26$ & $t_{83}=-2.34$ & $<0.05$ \\
\hline CES-D & $18.19 \pm 9.68$ & $13.79 \pm 7.51$ & $t_{77.76}=2.30$ & $<0.05$ & $15.69 \pm 8.35$ & $17.47 \pm 10.13$ & $t_{83}=-0.86$ & $<0.39$ \\
\hline $\mathrm{CES}-\mathrm{D} \geq 16$ & $52.1 \%$ & $36.4 \%$ & & $<0.12$ & $44.9 \%$ & $46.9 \%$ & & $<0.52$ \\
\hline Drive & $10.49 \pm 2.60$ & $10.89 \pm 2.89$ & $t_{83}=-0.67$ & $<0.51$ & $11.14 \pm 2.52$ & $9.97 \pm 2.86$ & $t_{83}=1.99$ & $<0.05$ \\
\hline Fun seeking & $10.16 \pm 2.50$ & $10.19 \pm 2.62$ & $t_{83}=-0.06$ & $<0.96$ & $10.44 \pm 2.45$ & $9.80 \pm 2.63$ & $t_{83}=1.15$ & $<0.25$ \\
\hline Reward responsiveness & $16.76 \pm 2.43$ & $16.97 \pm 2.25$ & $t_{83}=-0.42$ & $<0.68$ & $17.24 \pm 2.11$ & $16.29 \pm 2.57$ & $t_{83}=1.88$ & $<0.06$ \\
\hline BIS & $22.16 \pm 3.41$ & $22.53 \pm 3.20$ & $t_{83}=-0.50$ & $<0.62$ & $22.34 \pm 3.09$ & $22.29 \pm 3.64$ & $t_{83}=0.07$ & $<0.94$ \\
\hline
\end{tabular}

Groups with different severity of DWMH lesions differed in age $\left(t_{83}=-2.34 ; P<0.05\right)$ and on the mean scores of the drive dimension of the BIS/BAS scales $\left(t_{83}=1.99 ; P<0.05\right)$. Specifically, patients with more severe DWMHs were older $(53.89 \pm 13.26$ vs. $47.40 \pm$ 11.91) and reported lower scores on the drive dimension $(9.97 \pm 2.86$ vs. $11.14 \pm 2.52)$ than patients with mild or without any deep lesion.

The variables found to be significant at the bivariate level were inserted as independent variables in two generalized linear models with groups as dependent variables (see Table 4, 5). The models fit the data well (PWMH: Likelihood ratio $\chi_{1}^{2}=5.83 ; P<0.05$; DWMH: Likelihood ratio $\left.\chi_{2}^{2}=8.24 ; P<0.05\right)$. Patients with PWMHs were 1.06 times more likely to have lower CES-D scores $(P<0.05)$ than patients without PWMHs. Patients with more severe DWMHs were 1.04 times more likely to be older $(P<0.05)$ than patients with mild or without any DWMH lesions.

\section{Discussion}

Our aim was to assess whether WMHs were associated with depressive symptoms and sensitivity of the BIS and BAS systems in patients with chronic headache. Our findings suggest that differences in brain lesions are associated with differences in age and self-reported depression severity as measured by the CES-D.

Specifically, in multivariate analyses, individuals with PWMHs were more likely to report fewer depressive symptoms as compared with patients without PWMHs. These findings contradict our previous results regarding the association between white matter abnormalities and both unipolar and bipolar depression [8, 11, 14]. However, in our previous samples, we investigated patients with specific psychiatric conditions such as unipolar and bipolar disorders, whereas the current sample includes only individuals with chronic migraine without any evidence of comorbid psychiatric disorders. This may suggest that the association between WMHs and higher levels of depression is specific to particular subgroups of patients who have a psychiatric disorder $[11,12,14]$, and it may not be a factor among inpatients with medical conditions.

Whether the presence of WMHs predicts a poor prognosis in specific at-risk subgroups of patients has not been clearly established. Taylor et al. [44] found a relation between the progression and severity of WMHs and negative long-term outcomes in depressed patients. Prefrontal WMHs have been associated with cognitive dysfunctions in depressed older patients, poor or delayed antidepressant response [45], and poor treatment outcomes were associated with subcortical and basal ganglia, but not periventricular hyperintensities [46]. In contrast, there have been studies which reported no differences in treatment response and functional outcomes between elderly patients with and without WMHs [47, 48].

In our sample, those patients with more severe DWMHs differed from patients with mild or no DWMH lesions, as they were more likely to be older. Previous studies have strongly suggested that patients with migraine, particularly those diagnosed as having migraine with aura and at least 12 migraine episodes per year [49-51], are at an increased risk (with an odds ratio of 3.9) for subclinical brain infarctlike lesions, even after controlling for the most common cardiovascular risk factors and a history of cardiovascular diseases [52]. Unfortunately, we only examined the effect of age and did not consider whether patients with more pronounced cardiovascular risk factors were more likely to have DWMHs.

Although DWMHs have been suggested to have mainly a vascular origin, recent studies have reported that these brain lesions may reverse over time raising questions about their hypothetic ischemic origin [53, 54]. It has been demonstrated that frequent migraine attacks may be associated with increased iron concentration/accumulation in deep nuclei which is involved in central pain processing. These findings suggest that iron accumulation might have a 
Table 4 Generalized linear model: criterion PWMHs

\begin{tabular}{|c|c|c|c|c|c|c|c|c|c|c|}
\hline \multirow[t]{2}{*}{ Parameter } & \multirow[t]{2}{*}{$B$} & \multirow[t]{2}{*}{ Std. error } & \multicolumn{2}{|c|}{$95 \%$ Wald confidence interval } & \multicolumn{3}{|l|}{ Hypothesis test } & \multicolumn{3}{|c|}{$95 \% \mathrm{CI}$} \\
\hline & & & Lower & Upper & Wald Chi-square & $d f$ & Sig. & OR & Lower & Upper \\
\hline CES-D & -0.07 & 0.03 & -0.13 & -0.01 & 4.66 & 1 & 0.05 & 0.94 & 0.88 & 0.99 \\
\hline
\end{tabular}

Model fit: Likelihood ratio $\chi_{1}^{2}=5.83 ; P<0.05 ;$ AIC $=63.65 ;$ AICC $=63.81 ;$ Pearson $\chi_{31}^{2}=32.58 ;$ Value/g dl $=1.05$

Table 5 Generalized linear model: criterion DWMHs

\begin{tabular}{|c|c|c|c|c|c|c|c|c|c|c|}
\hline \multirow[t]{2}{*}{ Parameter } & \multirow[t]{2}{*}{$B$} & \multirow[t]{2}{*}{ Std. error } & \multicolumn{2}{|c|}{$95 \%$ Wald confidence interval } & \multicolumn{3}{|l|}{ Hypothesis test } & \multicolumn{3}{|c|}{$95 \% \mathrm{CI}$} \\
\hline & & & Lower & Upper & Wald Chi-square & $d f$ & Sig. & OR & Lower & Upper \\
\hline Drive & -0.16 & 0.09 & -0.33 & 0.02 & 3.15 & 1 & 0.08 & 0.86 & 0.72 & 1.02 \\
\hline Age & 0.04 & 0.02 & 0.01 & 0.08 & 5.23 & 1 & 0.05 & 1.04 & 1.01 & 1.08 \\
\hline
\end{tabular}

Model fit: Likelihood ratio $\chi_{2}^{2}=8.24 ; P=0.05 ; \mathrm{AIC}=109.02 ; \mathrm{AICC}=109.33$; Pearson $\chi_{77}^{2}=81.15 ;$ Value/g dl $=1.05$

possible role in determining chronic migraine [49]. Other hypotheses have been proposed to explain the existence of white matter abnormalities in migraineurs although definitive conclusions are still not established. It has been suggested that recurrent migraine attacks may affect vulnerable deep tissues penetrating arteries and contributing to local critical hypoperfusion leading to minor brain injury observed as white matter abnormalities. Other proposed mechanisms have been suggested including atherosclerotic insults associated with the presence of cardiovascular risk factors $[55,56]$, endothelial dysfunction (e.g., both endothelial activation and impaired vascular reactivity) [57-59], genetic risk factors which are common in migraine and stroke [60], drugs commonly used to treat headache, having vasoconstrictor activity [61], and cardiac dysfunctions such as patent foramen ovale. In addition, irrespective of whether endothelial dysfunction is linked to platelet aggregation, recurrent endothelial insults may lead to microvascular brain damage contributing to white matter lesions [62].

The present study must be considered in the light of the following limitations. The small sample size limits the generalizability of the current findings. In addition, our MRI study was limited by low spatial resolution and performed using only a $1.5 \mathrm{~T}$ MRI scanner. Studies using a $3 \mathrm{~T}$ MRI scanner having a higher resolution are able to detect a higher number and more precise extents of WMHs. An analysis quantifying total white matter lesion volume would strengthen the findings. Diffusion tensor imaging techniques may be more sensitive in detecting white matter abnormalities in association with mood disorders. Recently, some authors [63] have demonstrated "occult" brain damage in migraine patients using diffusion-weighted MRI, particularly tractography.
Furthermore, the Fazekas modified rating scale, which was used to measure the frequency and intensity of white matter lesions, is a quantitative lesion assessment method that may be limited since it is a visual rating scale less objective than many volumetric methods available. Also, we have not systematically assessed clinical disease course, age, gender, comorbidities, or history of substance abuse and dependence in the individuals recruited. In addition, some limitations regarding the utilization of the CES-D must be mentioned. Lack of consistency in some item responses in the measurement of depression suggests that response patterns may be affected by unique conceptualizations of depression among different ethnic groups [64]. Although the stability and applicability of the CES-D Scale with various ethnic groups have been widely assessed, few studies have addressed the cross-contextual generalizability of the CES-D Scale.

\section{Conclusion}

Overall, patients with chronic headache having PWMHs were more likely to have lower self-reported depression than those without PWMHs, and patients with more severe DWMHs were more likely to be older than those with less severe DWMHs. Further studies are needed to investigate the relation between progression of migraine and onset of new white matter lesions while considering the possible association between migraine attack rate and progression of lesion impairment [25], as well as the link between white matter lesions, major affective disorders, and migraine attacks over time. Also, functional impairment related to the presence of self-reported depression in patients with chronic migraine should be considered in 
terms of long-term cognitive changes. Importantly, different mechanisms may be considered in the emergence of WMHs and it is possible that WMHs may represent only the 'tip of the iceberg' in terms of structural white matter lesions.

\section{Conflict of interest None.}

Open Access This article is distributed under the terms of the Creative Commons Attribution License which permits any use, distribution, and reproduction in any medium, provided the original author(s) and the source are credited.

\section{References}

1. Thomas AJ, Perry R, Barber R et al (2002) Pathologies and pathological mechanisms for white matter hyperintensities in depression. Ann N Y Acad Sci 977:333-339

2. Thomas AJ, O'Brien JT, Davis S et al (2002) Ischemic basis for deep white matter hyperintensities in major depression: a neuropathological study. Arch Gen Psychiatry 59(9):785-792

3. Ovbiagele B, Saver JL (2006) Cerebral white matter hyperintensities on MRI: current concepts and therapeutic implications. Cerebrovasc Dis 22(2-3):83-90

4. Steffens DC, Krishnan KR (1998) Structural neuroimaging and mood disorders: recent findings, implications for classification, and future directions. Biol Psychiatry 43(10):705-712

5. Videbech P (1997) MRI findings in patients with affective disorder: a metaanalysis. Acta Psychiatr Scand 96(3):157-168

6. Ehrlich S, Noam GG, Lyoo IK et al (2004) White matter hyperintensities and their associations with suicidality in psychiatrically hospitalized children and adolescents. J Am Acad Child Adolesc Psychiatry 43(6):770-776

7. Ehrlich S, Breeze JL, Hesdorffer DC et al (2005) White matter hyperintensities and their association with suicidality in depressed young adults. J Affect Disord 86(2-3):281-287

8. Pompili M, Ehrlich S, De Pisa E et al (2007) White matter hyperintensities and their associations with suicidality in patients with major affective disorders. Eur Arch Psychiatry Clin Neurosci 257(8):494-499

9. Pompili M, Serafini G, Innamorati M et al (2010) White matter hyperintensities, suicide risk and late-onset affective disorders: an overview of the current literature. Clin Ter 161(6):555-563

10. Serafini G, Pompili M, Innamorati M et al (2010) Deep white matter hyperintensities as possible predictor of poor prognosis in a sample of patients with late-onset bipolar II disorder. Bipolar Disord 12(7):755-756

11. Serafini G, Pompili M, Innamorati M et al (2011) Affective temperamental profiles are associated with white matter hyperintensity and suicidal risk in patients with mood disorders. J Affect Disord 129(1-3):47-55

12. Grangeon MC, Seixas C, Quarantini LC et al (2010) White matter hyperintensities and their association with suicidality in major affective disorders: a meta-analysis of magnetic resonance imaging studies. CNS Spectr 15(6):375-381

13. Pompili M, Innamorati M, Mann JJ et al (2008) Periventricular white matter hyperintensities as predictors of suicide attempts in bipolar disorders and unipolar depression. Prog Neuropsychopharmacol Biol Psychiatry 32(6):1501-1507

14. Zanetti MV, Schaufelberger MS, de Castro CC et al (2008) White-matter hyperintensities in first-episode psychosis. $\mathrm{Br} \mathrm{J}$ Psychiatry 193(1):25-30
15. Taylor WD, Payne ME, Krishnan KR et al (2001) Evidence of white matter tract disruption in MRI hyperintensities. Biol Psychiatry 50(3):179-183

16. Soares JC, Mann JJ (1997) The anatomy of mood disordersreview of structural neuroimaging studies. Biol Psychiatry 41:86-106

17. Pompili M, Serafini G, Innamorati M et al (2010) Patient outcome in migraine prophylaxis: the role of psychopharmacological agents. Patient Relat Outcome Meas 1:107-118

18. Pompili M, Serafini G, Di Cosimo D et al (2010) Psychiatric comorbidity and suicide risk in patients with chronic migraine. Neuropsychiatr Dis Treat 6:81-91

19. Pompili M, Di Cosimo D, Innamorati M, Lester D, Tatarelli R, Martelletti P (2009) Psychiatric comorbidity in patients with chronic daily headache and migraine: a selective overview including personality traits and suicide risk. J Headache Pain 10(4):283-290

20. Fasmer OB, Oedegaard KJ (2001) Clinical characteristics of patients with major affective disorders and comorbid migraine. World J Biol Psychiatry 2:149-155

21. Breslau N, Schultz LR, Stewart WF et al (2000) Headache and major depression: is it the association specific to migraine? Neurology 54:308-313

22. Merikangas KR, Stevens DE, Angst J (1993) Headache and personality: results of a community sample of young adults. J Psychiatr Res 27:187-196

23. Merikangas KR, Merikangas JR, Angst J (1993) Headache syndromes and psychiatric disorders: association and family transmission. J Psychiatr Res 2:197-210

24. Puca F, the Italian Collaborative Group (2000) Psychological and social stressors and psychiatric comorbidity in patients with migraine without aura from headache centers in Italy a comparison with tension-type. J Headache Pain 1:17-32

25. Colombo B, Dalla Libera D, Comi G (2011) Brain white matter lesions in migraine: what's the meaning? Neurol Sci 32(Suppl 1):S37-S40

26. Schmitz N, Admiraal-Behloul F, Arkink EB (2008) Attack frequency and disease duration as indicators for brain damage in migraine. Headache 48(7):1044-1055

27. Gray JA (1994) Framework for a taxonomy of psychiatric disorder. In: Van Goozen HM, Van De Poll NE, Sergeant JA (eds) Emotions: Essays on emotion theory. Erlbaum, Hillsdale NJ, pp 29-59

28. Gray JA (1990) Brain systems that mediate both emotion and cognition. Special Issue: development of relationships between emotion and cognition. Cogn Emot 4:269-288

29. Mineka S, Watson D, Clark LA (1993) Comorbidity of anxiety and unipolar mood disorders. Ann Rev Psychol 49:377-412

30. Depue RA, Zald DH (1993) Biological and environmental processes in nonpsychotic psychopathology: A neurobehavioral perspective. In: Costello CG (ed) Basic issues in psychopathology. Guilford Press, New York, pp 127-237

31. Fowles DC (1993) Biological variables in psychopathology: A psychobiological perspective. In: Sutker PB, Adams HE (eds) Comprehensive handbook of psychopathology, 2nd edn. Plenum Press, New York, pp 57-82

32. Carver CS, White TL (1994) Behavioral inhibition, behavioral activation, and affective responses to impending reward and punishment: the BIS/BAS Scales. J Pers Soc Psychol 67:319-333

33. Jorm AF, Christensen H, Henderson AS, Jacom PA, Korten AE, Rodgers B (1999) Using the BIS/BAS scales to measure behavioural inhibition and behavioural activation: factor structure, validity and norms in a large community sample. Personality Individ Differ 26:49-58

34. Heubeck BG, Wilkinson RB, Cologon J (1998) A second look at Carver and White's (1994) BIS/BAS scales. Personality Individ Differ 25:785-800 
35. Beekman AT, Deeg DJ, Van Limbeek J, Braam AW, De Vries MZ, Van Tilburg W (1997) Criterion validity of the Center for Epidemiologic Studies Depression scale (CES-D): results from a community-based sample of older subjects in The Netherlands. Psychol Med 27:231-235

36. Somervell PD, Beals J, Kinzie JD, Boehnlein J, Leung P, Manson SM (1993) Criterion validity of the Center for Epidemiologic Studies Depression Scale in a population sample from an American Indian village. Psychiatry Res 47:255-266

37. Morin AJ, Moullec G, Maiano C, Layet L, Just JL, Ninot G (2011) Psychometric properties of the Center for Epidemiologic Studies Depression Scale (CES-D) in French clinical and nonclinical adults. Rev Epidemiol Sante Publique 59:327-340

38. Thomas JL, Jones GN, Scarinci IC, Mehan DJ, Brantley PJ (2001) The utility of the CES-D as a depression screening measure among low-income women attending primary care clinics. The Center for Epidemiologic Studies-Depression. Int J Psychiatry Med 31:25-40

39. Ros L, Latorre JM, Aguilar MJ, Serrano JP, Navarro B, Ricarte JJ (2011) Factor structure and psychometric properties of the center for epidemiologic studies depression scale (CES-D) in older populations with and without cognitive impairment. Int J Aging Hum Dev 72:83-110

40. Cheng CP, Yen CF, Ko CH, Yen JY (2011) Factor structure of the center for epidemiologic studies depression scale in Taiwanese adolescents. Compr Psychiatry 53(3):299-307

41. Wong WS, Chen PP, Yap J, Mak KH, Tam BK, Fielding R (2011) Assessing depression in patients with chronic pain: a comparison of three rating scales. J Affect Disord 133:179-187

42. Roberts RE, Vernon SW (1983) The Center for Epidemiologic Studies Depression Scale: its use in a community sample. Am J Psychiatry 140:41-46

43. Coffey CE, Wilkinson WE, Weiner RD et al (1993) Quantitative cerebral anatomy in depression. A controlled magnetic resonance imaging study. Arch Gen Psychiatry 50:7-16

44. Taylor WD, Steffens DC, MacFall JR et al (2003) White matter hyperintensity progression and late-life depression outcomes. Arch Gen Psychiatry 60:1090-1096

45. Kalayam B, Alexopoulos GS (1999) Prefrontal dysfunction and treatment response in geriatric depression. Arch Gen Psychiatry $56: 713-718$

46. Steffens DC, Bosworth HB, Provenzale JM, MacFall JR (2002) Subcortical white matter lesions and functional impairment in geriatric depression. Depr Anxiety 15:23-28

47. Salloway S, Boyle PA, Correia S et al (2002) The relationship of MRI subcortical hyperintensities to treatment response in a trial of sertraline in geriatric depressed outpatients. Am J Geriatr Psychiatry 10:107-111
48. Krishnan KR, Taylor WD, McQuoid DR et al (2004) Clinical characteristics of magnetic resonance imaging-defined subcortical ischemic depression. Biol Psychiatry 55:390-397

49. Kruit MC, van Buchem MA, Launer LJ et al (2010) Migraine is associated with an increased risk of deep white matter lesions, subclinical posterior circulation infarcts and brain iron accumulation: the population-based MRI CAMERA study. Cephalalgia 30:129-136

50. Kruit MC, Launer LJ, Ferrari MD, van Buchem MA (2006) Brain stem and cerebellar hyperintense lesions in migraine. Stroke 37:1109-1112

51. Kurth T, Tzourio C (2009) Migraine and cerebral infarct-like lesions on MRI: an observation, not a disease. JAMA 301: 2594-2595

52. Scher AI, Gudmundsson LS, Sigurdsson S et al (2009) Migraine headache in middle age and late-life brain infarcts. JAMA 301:2563-2570

53. Rozen TD (2007) Vanishing cerebellar infarcts in a migraine patient. Cephalalgia 27:557-560

54. Rozen TD (2010) Images from headache: white matter lesions of migraine are not static. Headache 50:305-306

55. Bousser MG, Welch KM (2005) Relation between migraine and stroke. Lancet Neurol 4:533-542

56. Welch KM (2003) Stroke and migraine-the spectrum of cause and effect. Funct Neurol 18:121-126

57. Elkind MS (2008) Endothelial repair capacity and migraine: the fix is in. Neurology 70:1506-1507

58. Lee ST, Chu K, Jung KH et al (2008) Decreased number and function of endothelial progenitor cells in patients with migraine. Neurology 70:1510-1517

59. Tietjen EG (2007) Migraine and ischaemic heart disease and stroke: potential mechanisms and treatment implications. Cephalalgia 27:981-987

60. Scher AI, Terwindt GM, Picavet HS et al (2005) Cardiovascular risk factors and migraine: the GEM population-based study. Neurology 64:614-620

61. Del Zotto E, Pezzini A, Giossi A et al (2008) Migraine and ischemic stroke: a debated question. J Cereb Blood Flow Metab 28:1399-1421

62. Tietjen GE (2009) Migraine as a systemic vasculopathy. Cephalalgia 29:987-996

63. Rocca MA, Pagani E, Colombo B et al (2008) Selective diffusion changes of the visual pathways in patients with migraine: a 3-T tractography study. Cephalalgia 28:1061-1068

64. Perreira KM, Deeb-Sossa N, Harris KM, Bollen K (2005) What are we measuring? An evaluation of the CES-D across race/ ethnicity and immigrant generation. Soc Forces 83:1567-1602 